\title{
The Effects on Ishikawa Endometrial Cancer Cell Lines upon Treatment with Hormonal Agents and Novel Drug Target
}

\author{
Pooja Pandita ${ }^{1}$, Xiyin Wang ${ }^{2}$, Shannon Hawkins ${ }^{2,3,4}$ \\ ${ }^{1}$ Indiana University School of Medicine, Indianapolis, IN, USA \\ ${ }^{2}$ Indiana University School of Medicine, Department of Obstetrics and \\ Gynecology, Indianapolis, IN, USA \\ ${ }^{3}$ Indiana University, Simon Cancer Center, Indianapolis, IN, USA \\ ${ }^{4}$ Indiana University, Department of Biochemistry and Molecular Biology, \\ Indianapolis, IN, USA
}

\begin{abstract}
Background and Hypothesis: Endometrial cancer is the most common gynecological malignancy in the US and will claim the lives of over 12,000 women in 2019. Women with high-risk histologic tumors, such as high-grade endometrial adenocarcinoma, represent over $50 \%$ of these deaths. Novel treatments are needed to treat these aggressive tumors. Decreased expression of the ribonuclease DICER is associated with high-grade and recurrent endometrial adenocarcinoma. To study the role of DICER in endometrial cancer, we used CRISPR/Cas9 to delete DICER in an endometrial cancer cell line. RNA sequencing revealed dysregulation of steroid hormone receptor signaling and high expression of APEX1 [apurinic/apyrimidinic endonuclease 1 (Ref-1/APE1)]. Elevated levels of Ref-1/APE1 have been found in various tumor types, and Ref1/APE1 inhibitors are in clinical trials. We hypothesize that our DICER deleted cell lines will be hormone insensitive yet respond to Ref-1/APE1 inhibitors by decreasing cellular proliferation.
\end{abstract}

Project Methods: Ingenuity Pathway Analysis provided an unbiased approach to determine dysregulated genes in steroid hormone signaling in our RNA transcriptomic data. QPCR was used to confirm gene expression in independent samples. Cells were treated with steroid hormones or Ref-1/APE1 inhibitors and proliferation was assessed using MTS.

Results: DICER deleted cell lines had downregulated expression of steroid hormone receptors. Treatment with steroid hormones did not have a significant effect on proliferation. However, treatment with Ref-1/APE1 inhibitors resulted in a significant decrease in proliferation.

Conclusion and Potential Impact: Ref-1/APE1 inhibitors affect cellular proliferation of endometrial cancer cell lines. Because survival has actually worsened for women with endometrial cancer, novel therapies such as Ref1/APE1 inhibitors deserve future study. 